\title{
CP Violation in Kaon Decay in the Scalar Strong Interaction Hadron Theory
}

\author{
F. C. Hoh \\ Dragarbrunnsg. 55C, Uppsala, Sweden \\ Email: hoh@telia.com
}

Received August 14, 2012; revised September 15, 2012; accepted September 22, 2012

\begin{abstract}
$\mathrm{CP}$ conservation and violation in neutral kaon decay are considered from a first principles' theory, recently published as "Scalar Strong Interaction Hadron Theory". The arbitrary phase angle relating $K^{0}$ and $\bar{K}^{0}$ in current phenomenology is identified to be related to the product of the relative energy to the relative time between the $s$ and $d$ quarks in these kaons. The argument of the $\mathrm{CP}$ violating parameter $\varepsilon$ is predicted to be $45^{\circ}$ without employing measured data. The $K_{S}^{0}$ decay rate is twice the $K_{L}^{0}-K_{S}^{0}$ masss difference, in near agreement with data, and both are proportional to the square of the relative energy $29.44 \mathrm{eV}$. Any pion from $K_{L}^{0}$ decay will also have a mass shift of $\approx 1.28 \times 10^{-5} \mathrm{eV}$. The present first principles' theory is consistent with CP conservation. To achieve CP violation, the relative time cannot extend to both $+\infty$ and $-\infty$ but is bounded in at least one direction. The values of these bounds lie outside the present theory and it is unknown how they can be brought forth. $\bar{B}^{0}-B^{0}$ mixing is also considered and the relative energy is $663.66 \mathrm{eV}$.
\end{abstract}

Keywords: CP Violation; Kaon Decay; Relative Energy; Relative Time; Scalar Strong Interaction

\section{Introduction}

$\mathrm{CP}$ vioaltion in neutral kaon decay has been treated phenomenologically [1-3] but its origin remains a mystery ever since its discovery nearly 50 years ago. This problem is now treated employing the recently developed first principles' theory [4] in which this mysterious origin is shown to be connected to the relative energy and relative time between the $\mathrm{s}$ and d quarks in the kaons.

Section 2 reproduces some phenomenological results. In Section 3, the arbitrary phase angle relating $\bar{K}^{0}$ to $K^{0}$ in Section 2 is identified to be connected to the relative energy and time among the quarks in the kaon. When applied to $K_{S}^{0} \rightarrow 2 \pi$, the phase of the CP violating parameter $\varepsilon$ in Section 2 is predicted by means of the degeneracies of $\mathrm{SU}(3)$ gauge fields to known $\mathrm{SU}(2)$ ones. The decay rate depends upon the relative energy. In Section 4 , the CP violating $K_{L}^{0} \rightarrow 2 \pi$ is found to be forbidden unless the relative time is bounded between certain finite values. The mass shift of $K_{L}^{0}$ depends upon the relative energy $29.44 \mathrm{eV}$ and is half the $K_{S}^{0} \rightarrow 2 \pi$ decay rate. Any pion from $K_{L}^{0}$ decay will also have a mass shift of $\approx 1.28 \times 10^{-5} \mathrm{eV}$. In Section 5 , the semileptonic decays of kaons are treated and $\mathrm{CP}$ violation also requires that the relative time be bounded in one direction. Section 6 summarizes the roles of relative energy and time and considers the possible origins of $\mathrm{CP}$ violation. $B^{0}-B^{0}$ mixing is similarly treated in Section 7 .

\section{Phenomenology $[2,3]$}

The decays $K_{S}^{0}, K_{L}^{0} \rightarrow 2 \pi, 3 \pi$ have been considered using the time-dependent Schrödinger equation without specifying the Hamiltonian in connection with CP nonconservation [2]. The starting point is the ansatz [2 (15.28)]

$$
\left|\bar{K}^{0}\right\rangle=\exp (\mathrm{i} S \Theta) C P T\left|K^{0}\right\rangle
$$

where $S$ denotes strangeness, $C P T$ the conventional discrete operator and $\Theta$ an arbitrary phase angle. Under $C P T$ invariance, [2] gives

$$
\begin{aligned}
& \left|K_{S}^{0}\right\rangle=\frac{1}{\sqrt{2}}\left(\begin{array}{c}
1+\varepsilon \\
1-\varepsilon
\end{array}\right), \\
& \left|K_{L}^{0}\right\rangle=\frac{1}{\sqrt{2}}\left(\begin{array}{c}
1+\varepsilon \\
-(1-\varepsilon)
\end{array}\right)
\end{aligned}
$$

where the upper row refers to $K^{0}$ and the low row to $\bar{K}^{0}$ and $\varepsilon$ is a small, complex quantity [2]. Using the measured [1] 


$$
\begin{aligned}
& m_{K L}-m_{K S}=3.483 \times 10^{-6} \mathrm{eV} \\
& \Gamma\left(K_{S}^{0} \rightarrow 2 \pi\right)=7.352 \times 10^{-6} \mathrm{eV} \approx 2\left(m_{K L}-m_{K S}\right)
\end{aligned}
$$

the argument of $\varepsilon \approx 45^{\circ}$ or $225^{\circ}$ has been deduced. Also, unitarity provides an upper bound for $|\varepsilon|$. Thus,

$$
\begin{aligned}
& \varepsilon \approx \pm|\varepsilon|(1+\mathrm{i}) / \sqrt{2}, \\
& |\varepsilon|<4.3 \times 10^{-3}
\end{aligned}
$$

Further, $(2.1,2)$ with $(7.2 .18)$ leads to

$$
2 \varepsilon \approx-\mathrm{i} \Theta
$$

so that the argument of $\Theta \approx-45^{\circ}$ or $-225^{\circ}$.

$[2(15.105,109,113)]$ define the ratio of the semileptonic decay ampitudes

$$
x=\frac{S_{f i}\left(\bar{K}^{0} \rightarrow \pi^{-} L_{s}^{+} v_{L}\right)}{S_{f i}\left(K^{0} \rightarrow \pi^{-} L_{s}^{+} v_{L}\right)}
$$

where $L$ stands for the lepton species, as in (7.1.11-13), and $s$ the lepton helicity. [2 $(15.116,117)]$ give the ratios of the decay rates to first order in $\varepsilon$,

$$
\begin{aligned}
\frac{\Gamma\left(K_{S}^{0} \rightarrow \pi^{-} L_{s}^{+} \nu_{L}\right)}{\Gamma\left(K_{S}^{0} \rightarrow \pi^{+} L_{-s}^{-} \bar{v}_{L}\right)} & =\left|\frac{1+\varepsilon+(1-\varepsilon) x}{(1+\varepsilon) x^{*}+(1-\varepsilon)}\right|^{2} \\
& \cong 1+4 \operatorname{Re}(\varepsilon) \\
& \cong \frac{\Gamma\left(K_{L}^{0} \rightarrow \pi^{-} L_{s}^{+} \nu_{L}\right)}{\Gamma\left(K_{L}^{0} \rightarrow \pi^{+} L_{-s}^{-} \bar{v}_{L}\right)}
\end{aligned}
$$

where $x$ turns out to be small and has been dropped.

From the measured semileptonic decay ratios [1], $(2.6 b, 4)$ yields

$$
\operatorname{Re}(\varepsilon)=1.612 \times 10^{-3}, \quad|\varepsilon| \approx 2.28 \times 10^{-3}
$$

Furthermore, [1] gives the nonleptonic CP violating amplitude ratios

$$
\begin{aligned}
& \left|\eta_{00}\right|=\sqrt{\frac{\Gamma\left(K_{L}^{0} \rightarrow \pi^{0} \pi^{0}\right)}{\Gamma\left(K_{s}^{0} \rightarrow \pi^{0} \pi^{0}\right)}}=2.221 \times 10^{-3} \\
& \left|\eta_{+-}\right|=\sqrt{\frac{\Gamma\left(K_{L}^{0} \rightarrow \pi^{+} \pi^{-}\right)}{\Gamma\left(K_{s}^{0} \rightarrow \pi^{+} \pi^{-}\right)}}=2.232 \times 10^{-3} \\
& \left(\delta^{a \dot{b}} \partial_{0}+\mathrm{i} \frac{1}{4} g \sqrt{2} W_{U}^{a \dot{b}}(X)\right)\left(\frac{1}{2}\left(-\delta^{b \dot{e}} \partial_{X 0}-\underline{\sigma}^{b \dot{e}} \partial_{\underline{X}}\right)-\underline{\sigma}^{b \dot{e}} \underline{\partial}\right) \frac{\chi_{0}(r)}{\sqrt{2}} \exp \left(-\mathrm{i} E_{0} X^{0}+\mathrm{i} \omega_{0} X^{0}\right) \\
& \chi_{0}(r)=\chi_{0}(\underline{x}),|\underline{x}|=r
\end{aligned}
$$

With the degeneracy (A15), (3.1) can be written as

$$
\left(\delta^{a \dot{b}} \mathrm{i} \omega_{0}+\mathrm{i} \frac{1}{4} g \frac{1+\mathrm{i}}{\sqrt{2}}\left(-\frac{Z^{a \dot{b}}(X)}{\cos \vartheta_{W}}\right)\right)\left(\frac{1}{2} \delta^{b \dot{e}} \mathrm{i} E_{0}-\underline{\sigma}^{b \dot{e}} \underline{\partial}\right) \frac{\chi_{0}(r)}{\sqrt{2}} \exp \left(-\mathrm{i} E_{0} X^{0}+\mathrm{i} \omega_{0} x^{0}\right)
$$


Since $\omega_{0}$ is generated by the weak interaction $g$ term, it must have the same phase in (3.2);

$$
\omega_{0}=(1+\mathrm{i}) \omega_{00}, \omega_{00}=\text { real constant }
$$

Turning to the first order term in the second operator in (7.1.9a) and repeat the above procedure. It is found that

$$
\omega_{0}=-(1+\mathrm{i}) \omega_{00}
$$

These two relations are related to the two phases in (2.4) but without using the empirical data (2.3); they are the consequences of the necessary degeneracy (A15). There is no conflict between (3.3) and (3.4) because they cancel out upon summing the two first order terms in (7.1.9a), as is reflected in (A8) which contains no linear $\omega_{0}$ term.

Follow now Section 7.3.2-4 and obtain the first order decay amplitude $S_{f i}\left(K_{S}^{0} \rightarrow 2 \pi\right)$. In the source term (7.3.5a), the first term in the braces is replaced by (3.2) multiplied by a final state wave function $\chi_{0(22)}^{+}$:

$$
\begin{aligned}
& \frac{\mathrm{i} g_{l}}{4} \chi_{0(r p) \dot{e} a}^{+}\left(\lambda_{l}\right)_{p s} W_{l}^{a \dot{b}}\left(\partial_{I I}^{f \dot{e}} \chi_{0(s r) b f}\right) \\
& \rightarrow \chi_{0(22) \dot{e} a}^{+} \times\left(\delta^{a \dot{b}} \mathrm{i} \omega_{0}+\mathrm{i} \frac{1}{4} g \frac{1+\mathrm{i}}{\sqrt{2}}\left(-\frac{Z^{a \dot{b}}(X)}{\cos \vartheta_{W}}\right)\right) \\
& \cdot\left(\frac{1}{2} \delta^{b \dot{e}} \mathrm{i} E_{0}-\underline{\sigma}^{b \dot{e}} \underline{\partial}\right) \frac{\chi_{0}(r)}{\sqrt{2}} \exp \left(-\mathrm{i} E_{0} X^{0}+\mathrm{i} \omega_{0} X^{0}\right)
\end{aligned}
$$

The final state $2 \pi$ comes from the decay of the $Z$ boson via $(7.7 .2,7)$ and the final state $\chi_{0(22)}^{+}$represents a vacuum meson state $\pi^{0}$ given by (7.3.20) with $\psi \rightarrow \chi$ which does not cntain any phase factor. Consider the integral over the relative time $x^{0}$ in the source $(7.3 .5 \mathrm{a})$ with the replacement (3.5),

$$
\begin{aligned}
& \int_{\mathrm{T}_{1}}^{\mathrm{T}_{2}} \mathrm{~d} x^{0} \exp \left(\mathrm{i} \omega_{0} x^{0}\right)=\int_{\mathrm{T}_{1}}^{\mathrm{T}_{2}} \mathrm{~d} x^{0} \exp \left((\mathrm{i}-1) \omega_{00} x^{0}\right) \\
& \mathrm{T}_{2}=-\mathrm{T}_{1}=\mathrm{T}_{0} \rightarrow \infty
\end{aligned}
$$

where (3.3) has been consulted. In the surface term (7.3.4), the exponential $\exp \left(\mathrm{i} \omega_{0} x^{0}\right)$ in (3.5) also enters but, in addition, also its complex conjugate $\exp \left(-\mathrm{i} \omega_{0}^{*} x^{0}\right)$ contained in $\chi_{0(23)}^{+}$in (7.3.4). The integral over the relative time $x^{0}$ in the surface integral (7.3.4) reads

$$
\begin{aligned}
& \int_{\mathrm{T}_{1}}^{\mathrm{T}_{2}} \mathrm{~d} x^{0} \exp \left(\mathrm{i} \omega_{0} x^{0}-\mathrm{i} \omega_{0}^{*} x^{0}\right) \\
& =\int_{\mathrm{T}_{1}}^{\mathrm{T}_{2}} \mathrm{~d} x^{0} \exp \left(-2 \omega_{00} x^{0}\right)
\end{aligned}
$$

Equations (7.3.4) and (7.3.5) are sandwiched between the initial stae $\left|i=K_{S}^{0}\right\rangle$ and final state $\langle f=0, Z=2 \pi|$ as was mentioned beneath (7.3.13). The 0 that follows $<$ $f=$ refers to the above-mentioned vacuum meson state $\pi^{0}$. By $(7.3 .3-5,13), S_{f i}\left(K_{S}^{0} \rightarrow 2 \pi\right)$ is proportional to the ratio of (3.6) to (3.7) which vanishes for $T_{0} \rightarrow \infty$ in (3.6);

$$
\begin{aligned}
& \frac{\int_{\mathrm{T}_{1}}^{\mathrm{T}_{2}} \mathrm{~d} x^{0} \exp \left(i \omega_{00} x^{0}-\omega_{00} x^{0}\right)}{\int_{\mathrm{T}_{1}}^{\mathrm{T}_{2}} \mathrm{~d} x^{0} \exp \left(-2 \omega_{00} x^{0}\right)} \\
& =-(1+\mathrm{i}) \frac{\exp \left((\mathrm{i}-1) \omega_{00} \mathrm{~T}_{2}\right)-\exp \left((\mathrm{i}-1) \omega_{00} \mathrm{~T}_{1}\right)}{\exp \left(-2 \omega_{00} \mathrm{~T}_{2}\right)-\exp \left(-2 \omega_{00} \mathrm{~T}_{1}\right)} \rightarrow 0
\end{aligned}
$$

This also holds if (3.3) is replaced by (3.4). Therefore, $K_{S}^{0} \rightarrow 2 \pi$ is forbidden to first order in $\mathrm{i} g W_{U}$. Even if this first order $S_{f i}\left(K_{S}^{0} \rightarrow 2 \pi\right)$ does not vanish, for instance by letting $T_{1}, T_{2}$ be finite such that (3.8) becomes of the magnitude of unity, it is expected to lead to a decay rate $\Gamma\left(K_{S}^{0} \rightarrow 2 \pi\right)$ of the same magnitude as $\Gamma\left(K^{ \pm} \rightarrow 2 \mathrm{p}\right) \cong 1.1 \times 10^{-8} \mathrm{eV}$ from phase space considerations. This rate is 668 times smaller than the observed rate in (2.3). This is "one of the remaining unsolved problems of the weak interaction" [2].

However, there is now a second order term $-\left(\omega_{0}\right)^{2}=$ $-\mathrm{i} 2\left(\omega_{00}\right)^{2}$ in (A8). Let $E_{00}$ be the $K^{0}$ mass in (A10) and $E_{0}$ $=E_{00}+\Delta E_{0 S}$ in (A8), the exponent in (3.5) will contain $-\mathrm{i} \Delta E_{0 S} X^{0}$ corresponding to the decay rate

$$
\Gamma\left(K_{S}^{0} \rightarrow 2 \pi\right)=\mathrm{i} \Delta E_{0 S}=-4 \omega_{00}^{2} / E_{00}
$$

where the lower sign in (A10) is chosen. Note that the relative energy $\omega_{00}$ is a hidden variable but its square in (3.9) is visible. Although (3.9) comes from a second order term, its amplitude is of first order in $\omega_{0}$, hence of order $g$. In (3.2), the ratio of $\omega_{0}$ to the $g$ term that leads to the last mentioned $S_{f i}\left(K_{S}^{0} \rightarrow 2 \pi\right)$ is not fixed or known. This allows $\Gamma\left(K_{S}^{0} \rightarrow 2 \pi\right)$ to be $\gg \Gamma\left(K^{ \pm} \rightarrow 2 \pi\right)$; it may be regarded that the nonvanishing $S_{f i}\left(K_{S}^{0} \rightarrow 2 \pi\right)$ in the last paragraph is greatly amplified by the relative energy $\omega_{00}$.

The factor $2 \approx \Gamma\left(K_{S}^{0} \rightarrow \pi^{-} \pi^{+}\right) / \Gamma\left(K_{S}^{0} \rightarrow 2 \pi^{0}\right)$ [1] comes from the factor $1 / \sqrt{2}$ in front of $\pi^{0}$ in (A5) that enters (7.7.2) via (7.7.7).

Instead of $\bar{K}^{0} \rightarrow \pi^{0} W_{U}$ of (7.3.27b) considered above, $K^{0} \rightarrow \bar{W}_{U} \pi^{0}$ of (7.3.29) can be treated in the same way. The only differences are that $(1+\mathrm{i}) \rightarrow(1-\mathrm{i})$ in $(3.3-4)$ and the upper sign in (A10) is used when deriving the corresponding (3.9); the above results remain unchanged.

If the vacuum meson state $\pi^{0}$ assigned to $\chi_{0(22)}^{+}$in (3.5) is replaced by a real final state $\pi^{0}, K_{S}^{0} \rightarrow 2 \pi$ there goes over to $K_{S}^{0} \rightarrow 3 \pi$. Because $\pi^{ \pm}$and $\pi^{0}$ form a triplet in the limit of SU(2) symmetry, they must have the same phase. Since $\pi^{ \pm}$cannot contain a phase factor with complex argument, $\exp \left((\mathrm{i}-1) \omega_{00} x^{0}\right)$, which would cause them to decay like $K_{s}^{0} \rightarrow 2 \pi$ in (3.9), this final state $\pi^{0}$ can at most contain a phase factor of the form $\exp \left(-\mathrm{i} \omega_{00} x^{0}\right)$, similar to that for $K_{L}^{0}$ following (4.6) below. The $\exp \left(-\omega_{00} x^{0}\right)$ factor in (3.6) remains unchanged and the ratio (3.8) remains $0 ; K_{S}^{0} \rightarrow 3 \pi$ is forbidden. However, if $T_{1} \rightarrow-T_{S}$ is finite, (3.8) becomes 
$2 \exp \left(-\omega_{00} T_{S}\right)$ and some $K_{S}^{0} \rightarrow 3 \pi$ will be seen. This form is the same as that for the semileptonic decay in (5.4) below. The difference is that $T_{K}$ in (5.4) given by (5.8) is small. Here, $T_{S}$ is much larger and is estimated to be $\sim 3 \times 10^{-15} \mathrm{sec}$ if the small $\Gamma\left(K_{S}^{0} \rightarrow 3 \pi\right) \approx 2.57 \times 10^{-12}$ $\mathrm{eV}[1]$ is to be accounted for.

\section{4. $K_{L}^{0} \rightarrow 2 \pi$, $3 \pi$ and $K_{L}^{0}-K_{S}^{0}$ Mass Difference}

The couplings $\bar{K}^{0} W_{U}$ and $\bar{W}_{U} K^{0}$ of (7.3.27b) and the second of (7.3.29) are the same with respect to $K_{S}^{0}$ but not to $K_{L}^{0}$ according to (7.2.18). This leads to results mentioned in the next to last paragraph of Section 3 for $K_{S}^{0}$. For $K_{L}^{0}$, the $W_{U}$ and $\bar{W}_{U}$ contributions to $K_{L}^{0} \rightarrow 2 \pi$ needs be summed, as has been done in revised $(7.3 .27,29)$ [unpublished]. The first order (3.1) for $K_{S}^{0}$ can be taken over here for $K_{L}^{0}$ if its sign is changed, as is seen in (7.2.18). Take the hermitian adjoint of (7.1.9a) and consider the first order term corresponding to (3.1) for $K_{L}^{0}$,

$$
\begin{aligned}
& \left(\delta^{b \dot{e}} \partial_{0}-\mathrm{i} \frac{1}{4} g \sqrt{2} \bar{W}_{U}^{b \dot{e}}(X)\right)\left(\frac{1}{2}\left(-\delta^{a \dot{b}} \partial_{X 0}-\underline{\sigma}^{a \dot{b}} \partial_{\underline{X}}\right)-\underline{\sigma}^{a \dot{b}} \underline{\partial}\right) \\
& \cdot \frac{\chi_{0}(r)}{\sqrt{2}} \exp \left(-\mathrm{i} E_{0} X^{0}+\mathrm{i} \omega_{0} X^{0}\right)
\end{aligned}
$$

Add this expression to the negative of (3.1) and work out the resulting expression using (A15-A16). The result shows that the imaginary part is of the form ig(real operator). Since $\partial_{0} \rightarrow \mathrm{i} \omega_{0}$ must have the same phase, analogous to that mentioned above (3.3), $\omega_{0}$ must be real here. The summation removes the imaginary parts in the above (real operator). The expressions $(3.3,4)$ are replaced by

$$
\begin{gathered}
\omega_{0}=\omega_{00} \\
\omega_{0}=-\omega_{00}
\end{gathered}
$$

respectively, for $K_{L}^{0} \rightarrow 2 \pi$. The above (real operator) contains both $W_{6}$ and $W_{7}$ which are the same by (A15A16).

The treatment of for $K_{S}^{0} \rightarrow 2 \pi$ in (3.3-5), using (4.2) instead, can be taken over and the ratio (3.8) is here replaced by

$$
\begin{gathered}
\frac{\int_{-T}^{\mathrm{T}} \mathrm{d} x^{0} \exp \left(\mathrm{i} \omega_{00} x^{0}\right)}{\int_{-\mathrm{T}}^{\mathrm{T}} \mathrm{d} x^{0} \exp \left(\omega_{00} x^{0}(\mathrm{i}-\mathrm{i})\right)}=\frac{\sin \left(\omega_{00} T\right)}{\omega_{00} T} \rightarrow 0 \\
T=\tau_{0} / 2 \rightarrow \infty
\end{gathered}
$$

where (7.3.16) has been noted. Thus, $K_{L}^{0} \rightarrow 2 \pi$, just like $K_{L}^{0} \rightarrow 2 \pi$, is also forbidden to first order in $i g W_{U}$. This result agrees with the requirement of $\mathrm{CP}$ invariance
[2]. Being a first principles' theory, consequences of conservation laws, including CP conservation, are included in the equations of motion (7.1.8-9).

Turning to the second order term $-\left(\omega_{0}\right)^{2}=-\left(\omega_{00}\right)^{2}$ in (A8) via (4.2). Let $E_{00}$ be the $K^{0}$ mass in (A10) and $E_{0}$ $=E_{00}+\Delta E_{0 L}$ in (A8), the equvalent of (3.9) becomes the mass shift

$$
\Delta E_{0 L}=2 \omega_{00}^{2} / E_{00}
$$

when the upper sign in. in (A10) is chosen. The square of the relative energy among the $s$ and $d$ quarks in the hidden space $x$ is visible in the laboratory space $X$ in form of the mass shift (4.4), just like it is in form of the decay rate (3.9). Again, (4.4) comes from the second order $-\left(\omega_{0}\right)^{2}$ in (A8) but its amplitude is of first order in $\omega_{0}$, hence of order $g$. Comparison of (4.4) to (3.9) yields

$$
\Gamma\left(K_{S}^{0} \rightarrow 2 \pi\right)=2 \Delta E_{0 L}
$$

which is $5.25 \%$ smaller than data (2.3). This discrepancy has been used to modify the argument $45^{\circ}$ in (2.4) to about $43.5^{\circ}$ [1-3]. Here, this $45^{\circ}$ cannot be changed due to the constraint $(3.3,4)$. This discrepancy cannot be accounted for in the present theory. Similar to the CP violation cases mentioned above, finite $T_{2}, T_{1}$ in (3.8) will render the first order $S_{f i}\left(K_{S}^{0} \rightarrow 2 \pi\right)$ mentioned below it to contribute and to reduce this discrepancy.

Using (2.3) for $\Delta E_{0 L}$, (4.4) gives the relative energy between the $s$ and $d$ quarks in neutral kaons,

$$
\omega_{00}= \pm 29.44 \mathrm{eV}
$$

The upper sign is used. The lower sign can also be used if it is accompanied by $x^{0} \rightarrow-x^{0}$, as is evident from the phase factor $\exp \left(\mathrm{i} \omega_{0} x^{0}\right)$ in $(3.1)$, noting $(3.3,4)$. This leads to that the $T \mathrm{~s}$ in (3.6), at the end of Sec. 3 and in (5.8) and (6.1) below also change sign.

If the vacuum meson state $\pi^{0}$ in (3.5) adapted for $K_{L}^{0}$ is changed to a real $\pi^{0}, K_{L}^{0} \rightarrow 2 \pi$, forbidden by (4.3) above, turns into $K_{S}^{0} \rightarrow 3 \pi$. This real final state $\pi^{0}$ must have a phase factor $\exp \left(\mathrm{i} \omega_{00} x^{0}\right)$ which is to be inserted into the upper integral in (4.3) to make this ratio to become unity, as is implicit in [4]. This implies that any $\pi^{0}$ from $K_{L}^{0}$ decay will also have a mass shift analogous to (4.4). According to the last paragraph of Section 3, $\pi^{ \pm}$ and $\pi^{0}$ have the same phase in the limit of SU(2) symmetry so that any $\pi^{ \pm}$from $K_{L}^{0}$ decay will also have a mass shift. With (4.6) and the $\pi$ masses [1], (4.4) yields the shifts

$$
\begin{aligned}
\Delta E_{\pi 0} & =1.284 \times 10^{-5} \mathrm{eV} \\
\Delta E_{\pi \pm} & =1.277 \times 10^{-5} \mathrm{eV}
\end{aligned}
$$

Although these shifts are far less than the error margin $0.35 \mathrm{kev}$ for the $\pi$ masses and are not observable, it signifies that there exists two different species of $\pi$ triplets 
with slightly different masses.

$K_{S}^{0} \rightarrow 3 \pi$ has been treated in [4] employing the null relative energy condition (A9). A relaxation of this condition here however does not affect the results obtained there.

To achieve CP violation, let $T \rightarrow T_{L} \neq \infty$ in (4.3), like those mentioned in Section 3 for $\Gamma\left(K_{L}^{0} \rightarrow 2 \pi, 3 \pi\right)$. For $T_{L}=0$, (4.3) becomes unity and $\Gamma\left(K_{L}^{0} \rightarrow 2 \pi\right)$ like $\Gamma\left(K_{S}^{0} \rightarrow 2 \pi\right)$ mentioned beneath $(3.8), \approx \Gamma\left(K^{ \pm} \rightarrow 2 \pi\right)$ from phase space considerations. This rate is much greater than data. To account for the mearsured $\Gamma\left(K_{L}^{0} \rightarrow 2 \pi\right), T_{L}$ is chosen such that (4.3) becomes of the magnitude $\operatorname{Re}\left(\eta_{00}, \eta_{+-}\right) \sqrt{668} \approx 0.0407$, where

$\operatorname{Re}\left(\eta_{00}, \eta_{+-}\right)$are given by (2.7-9) and 668 has been mentioned beneath (3.8). Thus, CP is correctly violated if

$$
\begin{aligned}
& \sin \left(\omega_{00} \mathrm{~T}_{L}\right) / \omega_{00} T_{L} \approx 0.0407 \\
& T_{L} \approx \pm 0.1025, \pm 0.226, \pm 0.712 \mathrm{eV}^{-1}
\end{aligned}
$$

where (4.6) has been used. The angles $\omega_{00} T_{L}$ are close to multiples of $\pi$. These relative times are very short, of the order of $10^{-16} \mathrm{sec}$. These values, like the finite $T$ s mentioned in Section 3, cannot be predicted from the present theory and have to come outside of it.

\section{Semileptonic Decay of $K_{L}^{0}$ and $K_{S}^{0}$}

The amplitude for $\bar{K}^{0} \rightarrow \pi^{+} W^{-}$is given by (7.3.27a) and for $K^{0} \rightarrow \pi^{-} W^{+}$by the first of (7.3.29). Subsequently, $W^{-} \rightarrow \pi^{+} L^{-} v_{L}$ and $W^{+} \rightarrow \pi L^{+} v_{L}$, where $L=\mu$, $e$. But the amplitudes for $\bar{K}^{0} \rightarrow \pi^{-} W^{+}$and

$K^{0} \rightarrow \pi^{+} W^{-}$do not follow from the internal index combinations in (7.3.18-19) and hence do not appear in (7.3.26-29). These decays are therefore forbidden, in agreement with the selection rule $\Delta S=\Delta Q$ [2]. This rule is a consequence of the present theory and has been verified by that $x$ of (2.6a) is consistent with 0 [1].

The amplitudes $(7.3 .27 \mathrm{a}, 29)$ assume the null relative energy condition (A9). The rates of these decays have not been evaluated because the pions are relativistic and their wave functions unknown; the coarse nonrelativistic (3.5.23), (7.7.13) used for $K_{L}^{0} \rightarrow 3 \pi$ is insufficient here and in other $K \rightarrow 2 \pi$ decays. Nevertheless, the forms of (7.3.27a) and the first of (7.3.29) are the same so that the decay rates $\Gamma\left(K^{0} \rightarrow \pi^{-} L^{+} v_{L}\right)$ and $\Gamma\left(\bar{K}^{0} \rightarrow \pi^{+} L^{-} v_{L}\right)$ are the same. By (7.2.18), $K_{S}^{0}$ and $K_{L}^{0}$ contain equal amount of $\bar{K}^{0}$ and $K^{0}$, they contribute about equally to these semileptonic decays. This is approximately verfied by data [1] and corresponds to the $\mathrm{CP}$ conserving part of these decays.

As was mentioned in the beginning of Section 3, (A9) has been relaxed here. Since the amplitudes for $\bar{K}^{0} \rightarrow \pi^{+} W^{-}$and $\bar{K}^{0} \rightarrow \pi^{0} W_{U}$ have the same form according to (7.3.27), the developments (3.1-4) can with some modifications be taken over. The expression corresponding to (3.1) reads

$$
\begin{aligned}
& \left(\delta^{a \dot{b}} \partial_{0}+\mathrm{i} \frac{1}{4} g \sqrt{2}\left(W_{1}^{a \dot{b}}(X) \mp \mathrm{i} W_{2}^{a \dot{b}}(X)\right)\right) \\
& \cdot\left(\frac{1}{2}\left(-\delta^{b \dot{e}} \partial_{X 0}-\underline{\sigma}^{b \dot{e}} \partial_{\underline{X}}\right)-\underline{\sigma}^{b \dot{e}} \underline{\partial}\right) \\
& \cdot \frac{\chi_{0}(r)}{\sqrt{2}} \exp \left(-\mathrm{i} E_{0} X^{0}+\mathrm{i} \omega_{0} x^{0}\right)
\end{aligned}
$$

where the degeneration (A14) for $W_{V}^{ \pm}$together with (A13) have been used. Analgous to $W_{6}(X)=W_{7}(X)$ in (A15-A16), $W_{1}(X)=W_{2}(X)$ here inasmuch as the both $W_{I}^{ \pm}(X)$ have the same $X$ dependence, so that the lower sign form of (5.1) turns to a form nearly the same as (3.2). Thus, (3.3) holds for $\bar{K}^{0} \rightarrow \pi^{+} W^{-}$. For $K^{0} \rightarrow \pi^{-} W^{+}$, the upper sign of (5.1) lead to

$$
\omega_{0}=(1-i) \omega_{00}
$$

The phase factor considerations for $K_{S}^{0} \rightarrow 3 \pi$ in the last paragraph of Sec. 3 can be taken over here. The phase factor $\exp \left(-\mathrm{i} \omega_{00} x^{0}\right)$ for the final state $\pi^{0}$ there can also be used for the final state $\pi^{+}$in $\bar{K}^{0} \rightarrow \pi^{+} W^{-}$ here. Such a factor is requried for in $K_{L}^{0} \rightarrow 3 \pi$ mentioned beneath (4.6). Inserting this factor into (3.6) turns (3.8) into

$$
\begin{aligned}
& \frac{\int_{T_{1}}^{T_{2}} d x^{0} \exp \left(-\omega_{00} x^{0}\right)}{\int_{T_{1}}^{T_{2}} d x^{0} \exp \left(-2 \omega_{00} x^{0}\right)} \\
& =2 \frac{\exp \left(-\omega_{00} T_{2}\right)-\exp \left(-\omega_{00} T_{1}\right)}{\exp \left(-2 \omega_{00} T_{2}\right)-\exp \left(-2 \omega_{00} T_{1}\right)} \rightarrow 0 \\
& T_{2}=-T_{1}=T_{0} \rightarrow \infty
\end{aligned}
$$

Analogous to that $K_{S}^{0} \rightarrow 2 \pi$ is forbidden by (3.8), the semileptonic decay $\bar{K}^{0} \rightarrow \pi^{+} W^{-} \rightarrow \pi^{+} L^{-} v_{L}$ is also forbidden when the relative energy $\omega_{00} \neq 0$, contrary to observation. If $\omega_{00}=0$ or the null relative energy condition (A9) holds, (5.3) turns into $\infty / \infty \rightarrow 1$ and these semileptonic decays can take place, as was implied in the second paragraph of this section. These decays however conserve $\mathrm{CP}$.

$\mathrm{CP}$ violation can be obtained in a way similar to that achieved in the last paragraph of Section 4, in which the limits of the relative time $x^{0}$ were not allowed to extend to $\pm \infty$. Let $T_{2}=T_{0}=\infty$ as in (5.3) and (3.6) but $T_{1}=-T_{K}$ finite, (5.3) turns into

$$
\frac{\int_{-\mathrm{T}_{K}}^{\infty} \mathrm{d} x^{0} \exp \left(-\omega_{00} x^{0}\right)}{\int_{-\mathrm{T}_{K}}^{\infty} \mathrm{d} x^{0} \exp \left(-2 \omega_{00} x^{0}\right)}=2 \exp \left(-\omega_{00} T_{K}\right)
$$

For $K^{0} \rightarrow \pi^{-} W^{+} \rightarrow \pi^{-} L^{+} v_{L}$, (5.2) replaces (3.3). Let $-T_{1}=T_{0}=\infty$ as in (5.3) and (3.6) but $T_{2}=-T_{K}$, the 
equivalent of (5.4) reads

$$
\frac{\int_{-\infty}^{-\mathrm{T}_{K}} \mathrm{~d} x^{0} \exp \left(\omega_{00} x^{0}\right)}{\int_{-\infty}^{-\mathrm{T}_{K}} \mathrm{~d} x^{0} \exp \left(2 \omega_{00} x^{0}\right)}=2 \exp \left(\omega_{00} T_{K}\right)
$$

As was mentioned in the second paragraph of this section, $K_{S}^{0}$ and $K_{L}^{0}$ contain equal amount of $K^{0}$ and $\bar{K}^{0}$ and $\Gamma\left(K^{0} \rightarrow \pi^{-} L^{+} v_{L}\right)=\Gamma\left(\bar{K}^{0} \rightarrow \pi^{+} L^{-} v_{L}\right)$ for the dominating $\mathrm{CP}$ conserving part. This together with (5.4-5) give the expression corressponding to (2.6b)

$$
\begin{aligned}
& \frac{\Gamma\left(K_{S}^{0} \rightarrow \pi^{-} L_{s}^{+} \nu_{L}\right)}{\Gamma\left(K_{S}^{0} \rightarrow \pi^{+} L_{-s}^{-} \bar{v}_{L}\right)}=\frac{\Gamma\left(K_{L}^{0} \rightarrow \pi^{-} L_{s}^{+} \nu_{L}\right)}{\Gamma\left(K_{L}^{0} \rightarrow \pi^{+} L_{-s}^{-} \bar{v}_{L}\right)} \\
& \approx\left|\frac{\exp \left(\omega_{00} T_{K}\right)}{\exp \left(-\omega_{00} T_{K}\right)}\right|^{2} \cong 1+4 \omega_{00} T_{K}
\end{aligned}
$$

Comparison with (2.6b) gives the identification

$$
\omega_{00} T_{K}=\operatorname{Re}(\varepsilon)
$$

This together with (2.7) and the lower of (4.6) yields

$$
T_{K} \approx 5.5 \times 10^{-5} \mathrm{eV}^{-1}=1.2 \times 10^{-11} \mathrm{~s}
$$

which is more than 10 times shorter than the $K_{S}^{0}$ decay time. Thus, by suitable choices of the limits of the relative times, the present theory reproduces the rather successful phenomenological (2.6b).

\section{On the Origin of CP Violation in Neutral Kaon Decay}

From the semileptonic decays in Section 5, the phenomenological phase angle $\Theta$ in (2.1) by (2.4-5) and (5.7) becomes

$$
\begin{aligned}
\Theta & =\mathrm{i} 2 \varepsilon \approx \pm \mathrm{i} \sqrt{2}|\varepsilon|(1+\mathrm{i}) \\
& = \pm \mathrm{i} 2 \omega_{00} T_{K} \mp 2 \omega_{00} T_{K}
\end{aligned}
$$

Here, $\Theta$ is identified with the product of a relative energy and a relative time, both finite. For the $\mathrm{CP}$ violating $K_{L}^{0} \rightarrow 2 \pi$ in Section 4 , the phase angle $\omega_{00} T_{L}$ in (4.8) via (4.3) associated with $K_{L}^{0}$ is real. This corresponds to that the angle $\Theta$ in (2.1) is also real for this case and is related to $\omega_{00} T_{L}$, also a product of a finite relative energy and a finite relative time.

Equations (5.4-5) show that it is the imaginary part of the relative energy $\omega_{0}$ in (3.3) and (5.2) that causes (5.6) to deviate from unity and thereby causes $\mathrm{CP}$ violation in the semileptonic decays, which takes place when the relative time $x^{0}$ between the $s$ and $d$ quarks does not extend to both $+\infty$ and $-\infty$ but is finite at one end.

This does not conflict with that $-\infty \leq x^{0} \leq \infty$ in (7.4.6b) via (7.3.16) to generate the $M_{W}$ mass. There, the actions in (7.4.3-4) involve stationary $K^{0}$ and $\bar{K}^{0}$ and not their decay so that (A9) can and has been applied in that stage. The phase factor $\exp \left(\mathrm{i} \omega_{0} x^{0}\right)$ here reduces to 1 there which allows for $-\infty \leq x^{0} \leq \infty$, similar to that $\omega_{00}=0$ in (5.3) ff renders it to be $\infty / \infty \neq 0$.

For the CP violating $K_{L}^{0} \rightarrow 2 \pi$, the relative time $x^{0}$ also does not extend to both $+\infty$ and $-\infty$ but is finite at both ends as is shown in (4.3) with $T \rightarrow T_{L}$ given in (4.8).

Summarizingly, CP conservation is related to the phase angle $\omega_{0} x^{0}$. The relative energy $\omega_{0}$ gives rise to the mass shifts for $K_{L}^{0}$ in (4.4) and pions in (4.7) and to that the large $K_{S}^{0} \rightarrow 2 \pi$ decay rate in (3.9) is twice the $K_{L}^{0}$ mass shift in (4.5). These results are derived within the frame work of the present theory.

The relative energy $\omega_{0}$ is necessary but not sufficient to account for the CP violating $K_{S}^{0} \rightarrow 2 \pi$ in Section 4 and the semileptonic decays in Section 5. To achieve these CP violations, bounds need be put on the values of the relative time $x^{0}$ so that it cannot run from $-\infty$ to $\infty$. The values of these bounds, given in (4.8) and (5.8), respectively, lie outside the present theory.

It not known how such relative time bounds can be brought forth. One observation is that the relative time $\left|x^{0}\right|=\left|x_{\mathrm{II}}^{0}-x_{\mathrm{I}}^{0}\right|$ must be shorter than twice the laboratory time $2 X^{0}=\left|x_{\mathrm{II}}^{0}+x_{\mathrm{I}}^{0}\right|$ in (3.1.3a). Now this $X^{0}$ is limited by the finite decay time so that the relative time is also limited. Another one is that the relative time $\left|y^{0}\right|$ has been set to the finite $2 / d_{m}$ in the last line of $\$ 7.7 .2$. Further, how the kaons are produced may also enter here. In any case, the $\mathrm{CP}$ violations here are not related to the $\mathrm{CP}$ violating phase in neutrino oscillation phenomenology [1 Neutrino Mixing].

In this connection, it may be pointed out that the boundary condition of the wave functions $\chi$ and $\psi$ in (A1), hence also in the action (7.1.8), at $\left|x^{0}\right| \rightarrow \infty$, like $T_{0}$ and $T$ in (3.6-7), (4.3) and (5.3), has been assumed to be the same as the corresponding ones (fixed) in the laboratory time $\left|x^{0}\right|$, as was pointed out in Section 6.1.1. The validity of this assumption has not been fully investigated and it is not clear whether this may impact upon the above relative time considerations.

\section{7. $\bar{B}^{0}-B^{0}$ Mixing}

The $\bar{B}^{0}-B^{0}$ mixing differs fundamentally from the $\bar{K}^{0}-K^{0}$ mixing considered above. In Table 1 , the $u, d$ and $s$ quarks have about the same mass. This leads to that $M_{m p r}^{2}$ in (2.4.1) differ by $\leq 12 \%$, as was mentioned above (2.4.5). Therefore, the kaons and pions are members of an approximate $\mathrm{SU}(3)$ octet. In the limit of SU(3) symmetry, the $u, d$ and $s$ quark masses coalesce. $\bar{K}^{0}$ and $K^{0}$ belong to the same octet providing basis vectors of the regular representation of $\mathrm{SU}(3)$. They can be transformed into each other by suitable choice of the 
Table 1. Quark masses and $d_{m_{0}}$ obtained from (5.1.1-4) using the masses $\pi^{+}, K^{+}, K^{0}, D^{0}, D_{s}^{+}$and $B^{0}$, and quark contents of [P1].

\begin{tabular}{cccccc}
\hline$m_{1}(\mathrm{GeV})$ & $m_{2}-m_{1}$ & $m_{3}$ & $m_{4}$ & $m_{5}$ & $d_{m_{0}}\left(\mathrm{GeV}^{2}\right)$ \\
\hline 0.6592 & 0.00215 & 0.7431 & 1.6215 & 4.7786 & 0.24455 \\
\hline
\end{tabular}

transformation $U_{3 q s}(X)$ of (7.1.7). Their linear combinations $K_{S}^{0}$ and $K_{L}^{0}$ in (7.2.18) are also vectors in this octet space on par with the pion vectors and represent physical mesons suitable for describing some weak decays.

The $c$ and $b$ qaurks are much heavier and the $D$ and $B$ mesons cannot meaningfully be accomodated in $\mathrm{SU}(4,5)$ multiplets together with the kaons and pions, as was indicated above (2.4.5). In the limit of SU(3) symmetry, $B^{0}$ belongs to the triplet $\left(B^{+}, B^{0}, B_{S}^{0}\right)$ providing basis vectors of the first fundamental representation of $\mathrm{SU}(3)$. But $\bar{B}^{0}$ belongs to the antitriplet $\left(B^{-}, \bar{B}^{0}, \bar{B}_{S}^{0}\right)$ providing basis vectors of the second fundamental representation of SU(3) transforming differently [6]. Thus, $B^{0}$ and $\bar{B}^{0}$ cannot be transformed into each other and linear combinations of them of the type (7.2.18) do not have definite transformation properties and hence are not members of any SU(n) multiplet. They remain as physical mesons in two different triplets. The $D$ meson triplets behave analogously.

As in Section 3, the null relative energy condition (A9) is also relaxed for $B^{0}$ and $\bar{B}^{0}$. The complex $\omega_{0}$ in (3.3) is due to the degeneration (A15) involving the complex neutral gauge boson $W_{U}$. Data [1] however show that the semileptonic decays of $B^{0}$ and $\bar{B}^{0}$ proceed dominantly via charged gauge bosons $W^{ \pm} \rightarrow$ charged leptons; the branching ratio of $B^{0} \rightarrow \bar{D}^{0} Z \rightarrow \bar{D}^{0}(2 K, 2 \pi)$ is very small. Therefore, $\omega_{0}=\omega_{00}=$ real as in (4.2) for $K_{L}$.

Therefore, a mass shift $\Delta E_{B^{0}}$ equivalent to $\Delta E_{0 L}$ in (4.4) also holds for $B^{0}$. For $\bar{B}^{0}$, the lower sign in (A10) is chosen. The laboratory time $X^{0}$ dependent part of the $B^{0}$ and $\bar{B}^{0}$ wave functions is

$$
\begin{aligned}
& \Psi_{B^{0}}\left(X^{0}\right)=\exp \left[\left(-\frac{\Gamma_{B^{0}}}{2}-\mathrm{i} E_{00}-\mathrm{i} \Delta E_{B^{0}}\right) X^{0}\right] \\
& \Psi_{\bar{B}^{0}}\left(X^{0}\right)=\exp \left[\left(-\frac{\Gamma_{B^{0}}}{2}-\mathrm{i} E_{00}+\mathrm{i} \Delta E_{B^{0}}\right) X^{0}\right]
\end{aligned}
$$

where $E_{00}$ is the mass and $\Gamma_{B^{0}}$ the decay rate of $B^{0}$ and $\bar{B}^{0}$. The $X^{0}$ dependent part of the probability of mixed $B^{0}$ and $\bar{B}^{0}$ is

$$
\begin{aligned}
& \left|\Psi_{B^{0}}\left(X^{0}\right) \pm \Psi_{\bar{B}^{0}}\left(X^{0}\right)\right|^{2} \\
& = \pm 2\left(\exp \left(-\Gamma_{B^{0}} X^{0}\right)\right)\left(1+\cos \left(2 \Delta E_{B^{0}} X^{0}\right)\right) \\
& \Delta E_{B^{0}}=2 \omega_{00}^{2} / E_{00}
\end{aligned}
$$

It is proportional to the phenomenological probability for remaining in the original bottom state [3]

$$
\begin{aligned}
& P\left(B^{0} \rightarrow B^{0}\right)=P\left(\bar{B}^{0} \rightarrow \bar{B}^{0}\right) \\
& =\frac{1}{2} \exp (-\Gamma t)\left(1+\cos \left(\Delta m_{d} t\right)\right)
\end{aligned}
$$

Here, $\Gamma=\Gamma_{B^{0}}, t=X^{0}$ and the oscillation frequency $\Delta m_{d}=2 \Delta E_{B^{0}} \stackrel{B^{0}}{=} 3.337 \times 10^{-4} \mathrm{eV}$ [1]. With the $B^{0}$ mass $E_{00}=5.2796 \mathrm{GeV}$, the second of (7.2) gives the relative energy $\omega_{00}=663.66 \mathrm{eV}$ between the $b$ and $d$ quarks. This value is 22.5 times greater than $29.44 \mathrm{eV}$ in (4.6) for that between the $s$ and $d$ quarks in $K^{0}$. Note that the ratio between the $B^{0}$ and $K^{0}$ masses is 10.6 , nearly half the above ratio.

The mass difference $3.337 \times 10^{-4} \mathrm{eV}$ is somewhat less than the $B^{0}$ decay rate $\Gamma_{B^{0}}=4.43 \times 10^{-4} \mathrm{eV}[1]$, similar to that the $K_{L}^{0}-K_{S}^{0}$ mass difference is about half of the $K_{S}^{0}$ decay rate in (2.3). These mass differences, apart from their values, are outcomes of the present theory, irrespective $\mathrm{CP}$ violation. Analogously, $\mathrm{CP}$ violation in the $\bar{B}^{0}-B^{0}$ system is also attributed to relative energy and time between the $b$ and $d$ quarks. Such a treatment however requires the knowledge of the amplitudes of 5 specific decays [3] and is beyond the scope of this paper.

In passing, it may be noted that while mixings of $d, s$ and $b$ quarks take place, the $c$ and $u$ quarks with charge $2 e / 3$ do not seem to mix, as $\bar{D}^{0}-D^{0}$ mixing is absent in [1].

\section{Conclusions}

In the standard model, quarks in meson decay are treated [3] largely as leptons are in QED. The effects of quark confinement in the relative space and of the relative time between the quarks are practically lost.

In the paper, the relative energy between the quarks gives rise to the mass shift between $K_{S}^{0}$ and $K_{L}^{0}$, equalling half the $K_{S}^{0}$ decay rate, and $\bar{B}^{0}-B^{0}$ mixing. Hadron spectra stem from the relative space [4] and the relative time generates the $W$ and $Z$ boson masses [4] without Higgs. Here, $\mathrm{CP}$ violation is achieved by limiting the relative time to certain regions.

\section{REFERENCES}

[1] J. Beringer, et al., "Particle Data Group," Physical Review D, Vol. 86, No. 1, 2012, Article ID: 010001. doi:10.1103/PhysRevD.86.010001

[2] T. D. Lee, "Particle Physics and an Introduction to Field Theory," Harwood Academic Publisher, Newark, 1981.

[3] K. Kleinknecht, "Uncovering CP Violation: Experimental Clarification in the Neutral K Meson and B Meson Systems," Springer, Berlin, 2003.

[4] F. C. Hoh, "Scalar Strong Interaction Hadron Theory," Nova Science Publishers, New York, 2011. 
https://www.novapublishers.com/catalog/product_info.ph p?products_id $=27069$

[5] F. C. Hoh, "Scalar Strong Interaction Hadron Theory (SSI)—Kaon and Pion Decay," In: C. J. Hong, Ed., The
Large Hadron Collider and Higgs Boson Research, Nova Science Publishers, New York, 2011, pp. 1-77.

[6] D. B. Lichtenberg, "Unitary Symmetry and Elementary Particles,” Academic Press, Waltham, 1978. 


\section{Appendix Relevant Equations from the Book [4]}

Parts of the book [4] often referred to are reproduced here. The references in form of $(x, y, z)$ or $\S x, y, z$ below refer to those in [4].

The starting point is the equations of motion for meson including internal coordinates $\xi$ given by $(2.3 .19,21,23$, 27),

$$
\begin{aligned}
& \partial_{\mathrm{I}}^{a \dot{b}} \partial_{\text {IIeef }} \chi_{\dot{b}}^{f}\left(x_{\mathrm{I}}, x_{\mathrm{II}}\right) \xi_{r}^{p}\left(z_{\mathrm{I}}, z_{\mathrm{II}}\right) \\
& +\left(M_{m}^{2}-\Phi_{m}\left(x_{\mathrm{I}}, x_{\mathrm{II}}\right)\right) \psi_{\dot{e}}^{a}\left(x_{\mathrm{I}}, x_{\mathrm{II}}\right) \xi_{r}^{p}\left(z_{\mathrm{I}}, z_{\mathrm{II}}\right)=0 \\
& \partial_{\mathrm{I} \dot{b} c} \partial_{\mathrm{II}}^{d \dot{e}} \psi_{\dot{e}}^{c}\left(x_{\mathrm{I}}, x_{\mathrm{II}}\right) \xi_{r}^{p}\left(z_{\mathrm{I}}, z_{\mathrm{II}}\right) \\
& +\left(M_{m}^{2}-\Phi_{m}\left(x_{\mathrm{I}}, x_{\mathrm{II}}\right)\right) \chi_{\dot{b}}^{d}\left(x_{\mathrm{I}}, x_{\mathrm{II}}\right) \xi_{r}^{p}\left(\mathrm{z}_{\mathrm{I}}, \mathrm{z}_{\mathrm{II}}\right)=0 \\
& \overline{\lceil\rceil}_{\mathrm{I}\rceil_{\mathrm{II}}} \Phi_{m}\left(x_{\mathrm{I}}, x_{\mathrm{II}}\right) \\
& =-\frac{1}{2} g_{s}^{4} \operatorname{Re} \psi^{b \dot{a}}\left(x_{\mathrm{I}}, x_{\mathrm{II}}\right) \chi_{\dot{a} b}^{*}\left(x_{\mathrm{I}}, x_{\mathrm{II}}\right) \\
& M_{m}=\left(m_{p}+m_{r}\right) / 2
\end{aligned}
$$

Here, $x_{\mathrm{I}}$ and $x_{\mathrm{II}}$ are the quark and antiquark coordinates, respectively, $\partial_{\mathrm{I}}=\partial / \partial x_{\mathrm{I}}, \partial_{\mathrm{II}}=\partial / \partial x_{\mathrm{II}}, \chi$ and $\psi$ are the meson wave functions with the spinor indices $a, b, \cdots$ running from 1 to $2, \Phi_{m}$ the scalar interquark potential, $p$ the quark flavor, $q$ the antiquark flavor, and $z_{\mathrm{I}}$ and $z_{\mathrm{II}}$ respecttively the internal coordinates for the quark and the antiquark in an abstract complex three dimensional space. $p$, $r=1,2$ and 3 refer to the $u, d$ and $s$ quarks, respectively. Because the quark masses $m_{p}$ and $m_{r}$ are different for different mesons, (A1) can, after cancelling out the $\xi$ functions be written in the form (2.4.3)

$$
\begin{aligned}
& \partial_{\mathrm{I}}^{a \dot{b}} \partial_{\mathrm{II}}^{f \dot{e}} \chi_{(p r) \dot{b} f}\left(x_{\mathrm{I}}, x_{\mathrm{II}}\right) \\
& -\left(M_{m(p r)}^{2}-\Phi_{m}\left(x_{\mathrm{I}}, x_{\mathrm{II}}\right)\right) \psi_{(p r)}^{a \dot{e}}\left(x_{\mathrm{I}}, x_{\mathrm{II}}\right)=0 \\
& \partial_{\mathrm{I} \dot{b} c} \partial_{\mathrm{II} \dot{e}} \psi_{(p r)}^{c \dot{e}}\left(x_{\mathrm{I}}, x_{\mathrm{II}}\right) \\
& -\left(M_{m(p r)}^{2}-\Phi_{m}\left(x_{\mathrm{I}}, x_{\mathrm{II}}\right)\right) \chi_{(p r) \dot{b} d}\left(x_{\mathrm{I}}, x_{\mathrm{II}}\right)=0
\end{aligned}
$$

where $(p r)$ indicates the dependence upon the quark flavors. The matrix form of $\psi$ is shown in (2.4.18),

$$
\psi_{(p r)} \rightarrow\left(\begin{array}{ccc}
\psi_{(11)} & \psi_{(12)} & \psi_{(13)} \\
\psi_{(21)} & \psi_{(22)} & \psi_{(23)} \\
\psi_{(31)} & \psi_{(32)} & \psi_{(33)}
\end{array}\right)-\frac{1}{3}\left(\psi_{(11)}+\psi_{(22)}+\psi_{(33)}\right)=\left(\begin{array}{ccc}
\frac{1}{\sqrt{2}} \psi_{(\pi 0)}+\frac{1}{\sqrt{6}} \psi_{(\eta)} & \psi_{(\pi+)} & \psi_{(K+)} \\
\psi_{(\pi-)} & -\frac{1}{\sqrt{2}} \psi_{(\pi 0)}+\frac{1}{\sqrt{6}} \psi_{(\eta)} & \psi_{(K 0)} \\
\psi_{(K-)} & \psi_{(\bar{K} 0)} & -\frac{2}{\sqrt{6}} \psi_{(\eta)}
\end{array}\right),
$$

$\psi \rightarrow \chi_{a b}$

Follow (3.1.3a) and (3.5.7) and introduce the relative and laboratory coordinates,

$$
\begin{aligned}
x^{\mu} & =x_{\mathrm{II}}^{\mu}-x_{\mathrm{I}}^{\mu}, X^{\mu} \\
& =\left(1-a_{m}\right) x_{\mathrm{I}}^{\mu}+a_{m} x_{\mathrm{II}}^{\mu}, a_{m}=1 / 2
\end{aligned}
$$

where the relative space time $x^{\mu}=\left(\underline{x}, x^{0}\right)$ are hidden variables [4]. The wave functions of a pseudosclar meson at rest reads $(3.1 .1,5,7,9)$

$$
\begin{aligned}
& \psi^{b \dot{e}}\left(x_{\mathrm{I}}, x_{\mathrm{II}}\right)=\delta^{b \dot{e}} \psi_{0}\left(x_{\mathrm{I}}, x_{\mathrm{II}}\right) \\
& =\delta^{b \dot{e}} \psi_{0}(\underline{x}) \exp \left(-\mathrm{i} E_{0} X^{0}+\mathrm{i} \omega_{0} x^{0}\right), \psi \rightarrow \chi
\end{aligned}
$$

where $E_{0}$ is the mass of the meson and $\omega_{0}$ the relative energy among the quarks. Inserting (A7) into (A4a) for a given $(p r)$ and taking the trace, one obtains from the first line of (3.1.8),

$$
\begin{gathered}
{\left[E_{0}^{2} / 4-\omega_{0}^{2}+\Delta\right] \chi_{0}(\underline{x})=\left(\Phi_{m}-M_{m}^{2}\right) \psi_{0}(\underline{x})} \\
\Delta=\partial^{2} / \partial \underline{x}^{2}
\end{gathered}
$$

The null relative energy condition (3.5.6)

$$
\omega_{0}=0
$$

With this ansatz, (A8) together with $\Phi_{m}$ obtained from $(3.2 .8,20)$ and $(3.4 .1),(4.3 .2),(4.4 .1)$ leads to the steady state meson mass $(5.1 .1)$

$$
E_{00}= \pm \sqrt{\left(m_{p}+m_{r}\right)^{2}-4 d_{m 0}-d_{m}^{2}}
$$

where $d_{m}=0.864 \mathrm{GeV}$ is given by (5.2.3) and $d_{m 0}$ and the quarks masses $m_{p}$ are given in Table 1.

In the presence of $\mathrm{SU}(3)$ gauge fields, the operators in (A4) are generalized according to $(7.1 .4,5)$ and $(7.2 .14)$,

$$
\begin{gathered}
\partial_{\mathrm{I}}^{a \dot{b}}=\frac{1}{2} \partial_{X}^{a \dot{b}}-\partial^{a \dot{b}} \rightarrow D_{\mathrm{I} p s}^{a \dot{b}}=\frac{1}{2}\left(\partial_{X}^{a \dot{b}} \delta_{p s}+\mathrm{i} \frac{1}{2} g\left(\lambda_{l}\right)_{p s} W_{l}^{a \dot{b}}(X)\right)-\partial^{a \dot{b}} \delta_{p s}=\partial_{\mathrm{I}}^{a \dot{b}} \delta_{p s}+\mathrm{i} \frac{1}{4} g\left(\lambda_{l}\right)_{p s} W_{l}^{a \dot{b}}(X) \\
\partial_{\mathrm{II}}^{f \dot{e}}=\frac{1}{2} \partial_{X}^{f \dot{e}}+\partial^{f \dot{e}} \rightarrow D_{\mathrm{II} p s}^{f \dot{e}}=\partial_{\mathrm{II}}^{f \dot{e}} \delta_{p s}+\mathrm{i} \frac{1}{4} g\left(\lambda_{l}\right)_{p s} W_{l}^{f \dot{e}}(X)
\end{gathered}
$$




$$
\begin{aligned}
& \mathrm{i} \frac{g}{2}\left(\lambda_{l}\right)_{p s} W_{l a b}(X) \rightarrow \mathrm{i} \frac{g_{l}}{2}\left(\lambda_{l}\right)_{p s} W_{l a b}(X) \\
&=\mathrm{i} \frac{g}{2}\left(\begin{array}{ccc}
2 A \sin \vartheta_{W}+Z \cos 2 \vartheta_{W} / \cos \vartheta_{W} & \sqrt{2} W_{I}^{-} & \left(g_{4-7} / g\right) \sqrt{2} W_{V}^{-} \\
\sqrt{2} W_{I}^{+} & -Z / \cos \vartheta_{W} & \left(g_{4-7} / g\right) \sqrt{2} W_{U} \\
\left(g_{4-7} / g\right) \sqrt{2} W_{V}^{+} & \left(g_{4-7} / g\right) \sqrt{2} \bar{W}_{U} & -2 A \sin \vartheta_{W}+2 Z \sin ^{2} \vartheta_{W} / \cos \vartheta_{W}
\end{array}\right) \\
& \sqrt{2} W_{I}^{ \pm}=W_{1} \pm \mathrm{i} W_{2}, \sqrt{2} W_{V}^{ \pm}=W_{4} \pm \mathrm{i} W_{5} \\
& \sqrt{2} W_{U}=W_{6}+\mathrm{i} W_{7}, \sqrt{2} \bar{W}_{U}=W_{6}-\mathrm{i} W_{7}
\end{aligned}
$$

where $A$ and $Z$ are gauge fields (7.2.1), $\vartheta_{w}$ the Weinberg angle $(7.2,12)$ and $g$ and $g_{4-7}$ weak coupling constants (7.2.7).

There are four known gauge fields $W_{I}^{ \pm}, Z$ and $A$ in (7.2.2) but eight $W_{I}^{ \pm}, W_{3}, W_{8}, W_{V}^{ \pm}, W_{U}^{1}$, and $\bar{W}_{U}$ in (7.1.5). The last four gauge fields are new, have not been observed and are converted into the four known ones via the following degeneracy scheme Section 7.2.3. Thus, (7.2.20) reads

$$
\frac{g_{4-7}}{g} \sqrt{2} W_{\mathrm{V} a b}^{ \pm}(X) \rightarrow \sqrt{2} W_{\mathrm{I} a b}^{ \pm}(X)
$$

Equations $(7.2 .21,22)$ have been written down heuristically and are now replaced by the formal analog of (A14) as is evident from (A12),

$$
\begin{aligned}
& \frac{g_{4-7}}{g} \sqrt{2} W_{U}=\frac{g_{4-7}}{g}\left(W_{6}+\mathrm{i} W_{7}\right) \rightarrow \frac{1+\mathrm{i}}{\sqrt{2}}\left(-\frac{Z}{\cos \vartheta_{W}}\right) \\
& \frac{g_{4-7}}{g} \sqrt{2} \bar{W}_{U}=\frac{g_{4-7}}{g}\left(W_{6}-\mathrm{i} W_{7}\right) \rightarrow \frac{1-\mathrm{i}}{\sqrt{2}}\left(-\frac{Z}{\cos \vartheta_{W}}\right)
\end{aligned}
$$

where the magnitudes on both sides are equal. 